\title{
Potential therapeutic role of osteopontin in alcoholic liver disease
}

A recent study published in Hepatology demonstrated that mice overexpressing osteopontin in hepatocytes had less steatosis, inflammation and liver injury compared with wild-type mice and osteopontin knockout mice when fed the ethanol Lieber-DeCarli diet. The authors suggest that increasing the secretion of osteopontin and/or its excretion to the bile and faeces or oral administration of milk osteopontin could have therapeutic potential in alcoholic liver disease (ALD).

Osteopontin is induced in patients with alcoholism, but its role in the pathophysiology of ALD remains unclear. "Preliminary studies supporting this project indicated that dietary supplementation with milk osteopontin protected wild-type mice from chronic alcohol-induced liver injury by restoring tight junction protein expression," explains Natalia Nieto, corresponding author. The researchers wanted to take the project further by investigating whether osteopontin has a therapeutic role in
4 ...milk osteopontin could be an efficient, accessible, simple and inexpensive nutritional therapeutic approach... 77

slowing or stopping the progression of alcoholic hepatitis once it has started.

The authors investigated the effect of chronic alcohol feeding on wildtype mice, osteopontin knockout mice and transgenic mice overexpressing osteopontin in hepatocytes. Results from the study show that alcohol feeding leads to increases in levels of hepatic, plasma, biliary and faecal osteopontin. Notably, these levels were increased more in the transgenic mice than the wild-type mice. Mice overexpressing osteopontin also had less steatosis and less inflammation and liver injury (shown by reduced ALT activity, lipopolysaccharide [LPS] levels and numbers of macrophages, as well as other parameters) than wild-type and osteopontin knockout mice.
The researchers carried out binding studies and found that osteopontin showed affinity for LPS, and that this binding prevented macrophage activation, reactive oxygen and nitrogen species generation and TNF production. In addition, administration of milk osteopontin was found to block LPS translocation in vivo and to protect from early alcohol-induced liver injury.

"This work could affect clinical practice and have significant socioeconomic impact in the near future because dietary administration of milk osteopontin could be an efficient, accessible, simple and inexpensive nutritional therapeutic approach for slowing down and/or preventing the progression of ALD," concludes Nieto.

Isobel Leake 